Methods: 66 patients with chronic low back pain were enrolled in this study. The patients were randomised and divided into two groups. Lomber stabilisation exercises, TENS and Hotpack treatments were given to the first group ( $\mathrm{n}: 28)$ while the second group ( $\mathrm{n}$ : 37 ) were treated with only TENS and Hotpack. Patients' evaluations have been carried out before and after 8 weeks following the exercises. Visuel Analogue Scale (VAS) were used to determine the pain as a numeric scale during the rest and pyhsical activity. Modified Oswestry Questionnaire Survey and Roland Morris Scale were used to evaluate of functional disability. The Short Form-36 (SF36) applied to measure of quality of life. Tetrax device has been used to evaluation the body balance. 7-15 MHZ Ultrasound has been used to evaluate the change of trophic changes of multifidus muscle's cross-sectional areas.

Results: Except falling risk, in all parameters we have observed significant improvement in group 1. Cross-sectional area of the multifidus muscles, physical role of SF-36, mental health and energy/vitality sub parameters were found significantly better in group 1 when compared to the other group. There was no significant difference between the groups when the fall index, VAS scores, Roland Morris Questionnaire and Oswestry Disability Index were considered.

Conclusions: As a result, lomber stabilisation exercises have positive effect on pain relief, improve the functional capacity and quality of life. Additionally the exercises also had benefit and increased cross-sectional areas of multifidus muscles. Disclosure of Interest: None declared

DOI: 10.1136/annrheumdis-2018-eular.1519

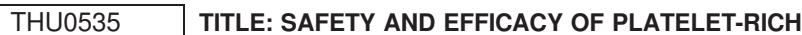 PLASMA IN TREATMENT OF CARPAL TUNNEL SYNDROME; A RANDOMISED CONTROLLED TRIAL}

L. Bagherzadeh, S.A. Raeissadat, A. Karimzadeh, M. Hashemi. Physical medicine and rehabilitation, shahid beheshti university of medical sciences, ehran, Iran, Islamic Republic of Ireland

Background: Carpal tunnel syndrome is the most common peripheral entrapment neuropathy, for which conservative treatments are the first measures taken but they are not usually sufficient.

Objectives: Recently major attention has been drawn to platelet-rich plasma, for its possible effects on axon regeneration and neurological recovery. Although few studies have evaluated the effects of this treatment in carpal tunnel syndrome, further investigations are required to reach concrete conclusion.

Methods: In this randomised controlled trial, women referring to the physical medicine and rehabilitation clinic at Shahid Modarres Hospital during 2016 with a diagnosis of mild and moderate idiopathic carpal tunnel syndrome were included and randomly assigned to two groups of control, only using a wrist splint, and platelet-rich plasma that received wrist splints along with a single local injection of platelet-rich plasma. The outcome measures were assessed via Visual Analogue Scale, the Boston Carpal Tunnel Syndrome Questionnaire and electrophysiological findings including the peak latency of sensory nerve action potential and the onset latency of the compound muscle action potential.

Results: A total of 41 women were included and randomly assigned to two groups of control (20 wrists) and platelet-rich plasma ( 21 wrists). Before treatment there were no significant differences between the two groups except for the median peak latency of sensory nerve action potential which was significantly higher among the patients in the platelet-rich plasma group $(p=0.03)$. All the measured variables significantly decreased in both groups after 10 weeks of treatment except for the median onset latency of the compound muscle action potential $(p=0.472)$. Finally, the changes in neither of the outcome measures evaluated were found to significantly differ between the two groups, even when the analyses were adjusted for age of the patients.

Conclusions: The findings of this study showed that in a relatively short period of time after treatment, a single injection of platelet-rich plasma in the wrist does not add significantly to the effects of conservative treatment with wrist splints, in regards to the women pain and symptom severity, functional status and electrophysiological parameters.

Disclosure of Interest: None declared

DOI: 10.1136/annrheumdis-2018-eular.5628

\section{THU0536 \\ KNOWLEDGE OF AND EXPECTATIONS ABOUT FUNCTIONAL RESTORATION PROGRAM FOR CHRONIC LOW BACK PAIN ARE OFTEN FALSE: A MIRROR} SURVEY OF 40 PATIENTS AND 59 PHYSICIANS IN 2017

L. Pierre $^{1}$, F. Bailly ${ }^{1}$, M. Assadourian ${ }^{1}$, V. Foltz ${ }^{1}$, A. Potel ${ }^{1}$, L. Morardet ${ }^{1}$, S. Ascione ${ }^{1}$, M. Le Ralle ${ }^{1}$, A. Dupeyron ${ }^{2}$, N. Ibrahim ${ }^{3}$, I. Griffoul-Espitalier ${ }^{4}$ B. Duplan ${ }^{5}$, J. Beaudreuil ${ }^{6}$, B. Fautrel ${ }^{1}$, L. Gossec ${ }^{1}$. ${ }^{1}$ Sorbonne university; ${ }^{2}$ Nimes hospital; ${ }^{3}$ Orleans hospital; ${ }^{4}$ Tours hospital; ${ }^{5}$ Metropole Savoie hospital;

${ }^{6}$ Lariboisiere hospital, Paris, France

Background: Functional restoration programs (FRP) are multi-disciplinary programs that have demonstrated their effectiveness in chronic low back pain (CLBP) but they appear to be little known by patients and/or physicians.

Objectives: To assess the knowledge and expectations of CLBP patients and physicians about FRP.

Methods: Multicenter cross-sectional study in 2017 in 6 tertiary-care hospitals in France. Consecutive patients with CLBP (more than 6 weeks) who were seen for their CLBP were included. Physicians (rheumatologists or general practitioners, GPs) who had referred at least 5 patients to the tertiary-care centres were sent a survey. Patients and physicians were asked about their knowledge and expectations of a FRP. Results were scored as 'correct' regarding health professionals involved in the FRP, if both doctors and (physiotherapists or physical activity coach) were ticked; and 'correct' for duration if 3 to 6 weeks. Expectations regarding the FRP were rated on a list of potential expectations on a scale of $0-10$. Expectations were scored ' correct' if return to work was scored $\geq 7 / 10$, and 'fully correct' if physical activity and pain management, but not 'healing' were also expected. Responses were then compared between patients and physicians by Chi 2 test.

Results: Of 172 patients, median age 48 years (IQR 38-58), median CLBP duration 5 years (IQR 2-10), 60.0\% women: 110 (64.0\%) had no knowledge of FRP 22 had already participated in an FRP. The remaining 40 patients were compared to physicians (figure 1 ). Of 80 physicians, $42.5 \%$ GPs and $53.7 \%$ rheumatologists, $57.5 \%$ women: 21 (26.2\%) had no knowledge of FRP ( $p$ value $<0.0001$ vs patients). The remaining 59 were compared to the 40 patients.

Knowledge of FRP health professionals was 'correct' for $13 / 40$ (32.5\%) patients vs $41 / 59(69.5 \%)$ physicians ( $p=0.0003)$; and knowledge of duration was 'correct' for $26 / 40(65.0 \%)$ vs $46 / 59(78.0 \%)$ physicians ( $p=0.15)$.

Expectations were 'correct' for $21 / 40(52.5 \%)$ patients vs $44 / 59(74.6 \%)$ for physicians $(p=0.02)$, and 'fully correct' for $9 / 40(22.5 \%)$ patients vs $16 / 59(27.1 \%)$ for physicians $(p=0.6)$.

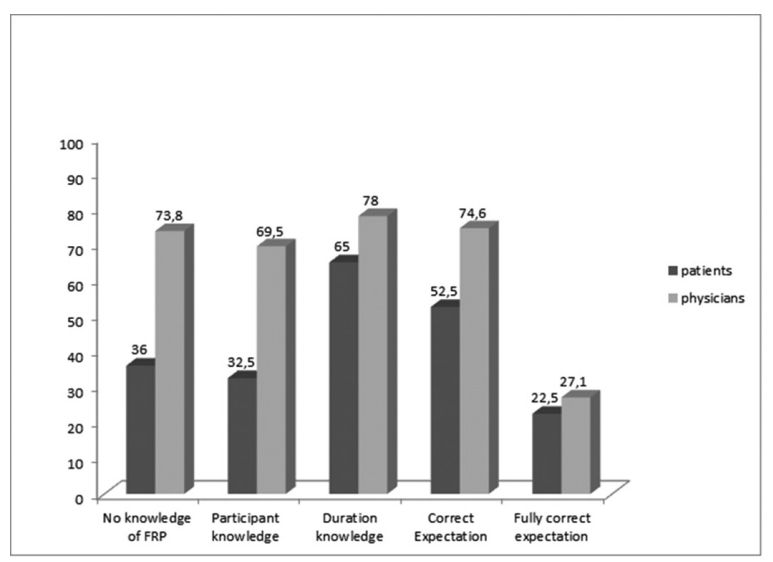

Conclusions: FRP was largely unknown to patients with CLBP. Even those who were aware of the FRP did not know what to expect. While physicians' knowledge and perception of the FRP was better, there was still a lack of knowledge and therefore a likely underutilization or misuse of the FRP. Information campaigns are needed.

Disclosure of Interest: None declared

DOI: 10.1136/annrheumdis-2018-eular.3754 